\title{
Newly designed extracardiac direct total cavopulmonary connection with merged connection and mixing route
}

\author{
Nobuyuki Ishibashi, MD, ${ }^{\text {a }}$ Mitsuru Aoki, MD, ${ }^{a}$ Manabu Watanabe, MD, ${ }^{a}$ Hiromichi Nakajima, MD,
}

Hiroyuki Aotsuka, MD, ${ }^{\text {b }}$ and Tadashi Fujiwara, MD, ${ }^{a}$ Chiba, Japan

I n total cavopulmonary connection (TCPC) streamlined cavopulmonary anatomic designs with minimum energy losses and an even flow to bilateral lungs are important to obtain optimal clinical results. However, in the conventional anatomic design of TCPC, it is difficult to obtain a good energy efficiency with balanced inferior vena caval (IVC) flow distribution. In 2003, using a computational fluid dynamics model, the lateral tunnel Fontan operation after the hemi-Fontan procedure was demonstrated to have the advantages of a good energy efficiency and a balanced distribution of the IVC flow based on the presence of a divergent shape in both the superior vena cava (SVC) and IVC anastomoses, the anteroposterior offset of the 2 caval anastomoses, and complete mixing in the right atrium. ${ }^{1}$ We agreed with this explanation but considered that there was a risk of development of right atrial dilation and late arrhythmia.

From the Departments of Cardiovascular Surgery ${ }^{\mathrm{a}}$ and Cardiology, ${ }^{\mathrm{b}}$ Chiba Children's Hospital, Chiba, Japan.

Received for publication Feb 11, 2006; accepted for publication Feb 20, 2006.

Address for reprints: Nobuyuki Ishibashi, MD, Departments of Cardiovascular Surgery, Heart Institute of Japan, Tokyo Women's Medical University, 8-1, Kawada-cho, Shinjuku-ku, Tokyo, 162-8666, Japan (E-mail: nobishibashi-ths@umin.ac.jp).

J Thorac Cardiovasc Surg 2006;132:162-3

$0022-5223 / \$ 32.00$

Copyright () 2006 by The American Association for Thoracic Surgery doi:10.1016/j.jtcvs.2006.02.039
Based on the above results, we newly designed and performed TCPC with the merged connection of the superior and inferior venous flow through direct anastomosis of the SVC with the IVC and a mixing route using the main pulmonary artery (PA). In this report we describe and discuss our procedure.

\section{Clinical Summary}

The patient was a 2-year-old girl who underwent a left modified Blalock-Taussig shunt at the age of 6 months under a diagnosis of double-inlet right ventricle and pulmonary stenosis. Cardiac catheterization at the age of 1 year and 7 months revealed a mean PA pressure of $15 \mathrm{~mm} \mathrm{Hg}$ and a pulmonary resistance of 1.0 wood/ unit. The PA trunk was well developed (Figure 1, A).

We proceeded to surgical intervention with cardiopulmonary bypass. The main PA and its branches and SVC were dissected, and the shunt was divided. The PA trunk, the IVC, and the SVC were divided at the sinus of Valsalva, the right atrial junction, and just a few millimeters distal to the right atrial junction, respectively. The right lateral wall of the distal stumps of the SVC and IVC were directly sutured, and this anastomosis was designed as a merged connection of the superior and inferior venous flow (Figure $1, B)$. Then the distal end of the PA trunk was sutured directly in an end-to-side fashion (Figure 1, C).

The postoperative clinical course was uneventful. At 3 months after the operation, catheterization showed PA, IVC, and SVC pressures of $10 \mathrm{~mm} \mathrm{Hg}$ each. Angiography showed a smooth and merged connection of the superior and inferior venous flow and a balanced flow distribution to the PA (Figure 2). Technetium ${ }^{99 m}$ scintigraphy revealed an almost balanced IVC flow distribution.

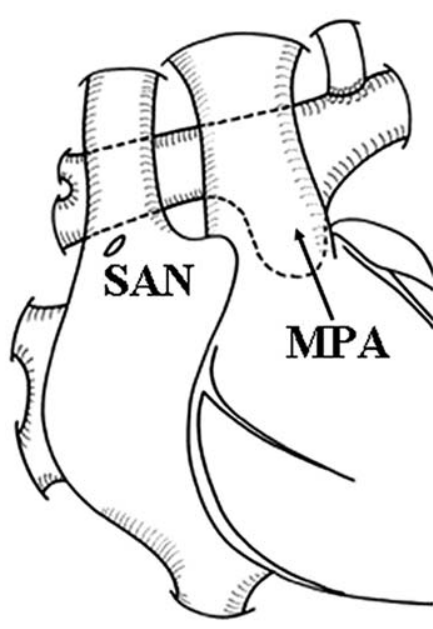

$\mathbf{A}$

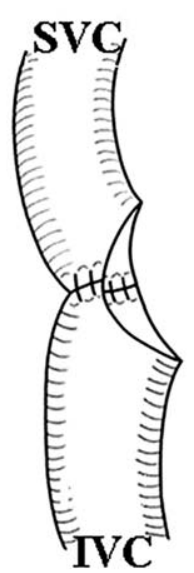

B

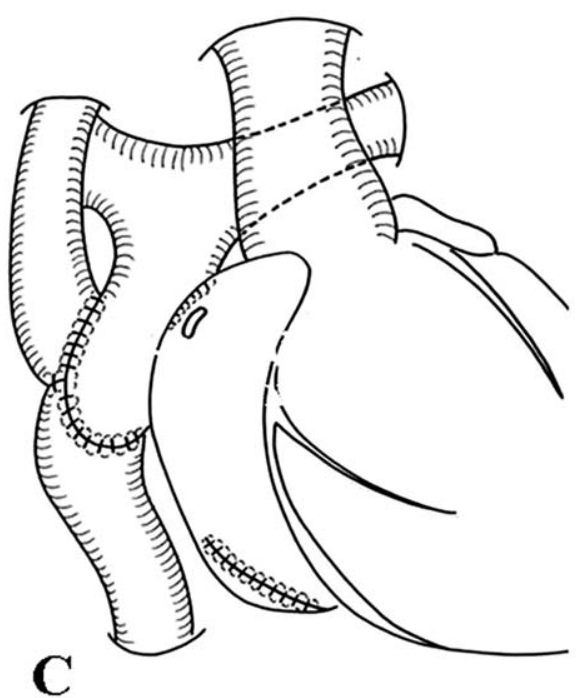

Figure 1. Operative scheme: $A$, before the operation; $B$, direct anastomosis of the right lateral wall of the superior vena cava (SVC) and inferior vena cava (IVC); C, extracardiac direct total cavopulmonary connection with the laminar combination of SVC and IVC flow and mixing route using the main pulmonary artery. SAN, Sinoatrial node; MPA, main pulmonary artery. 


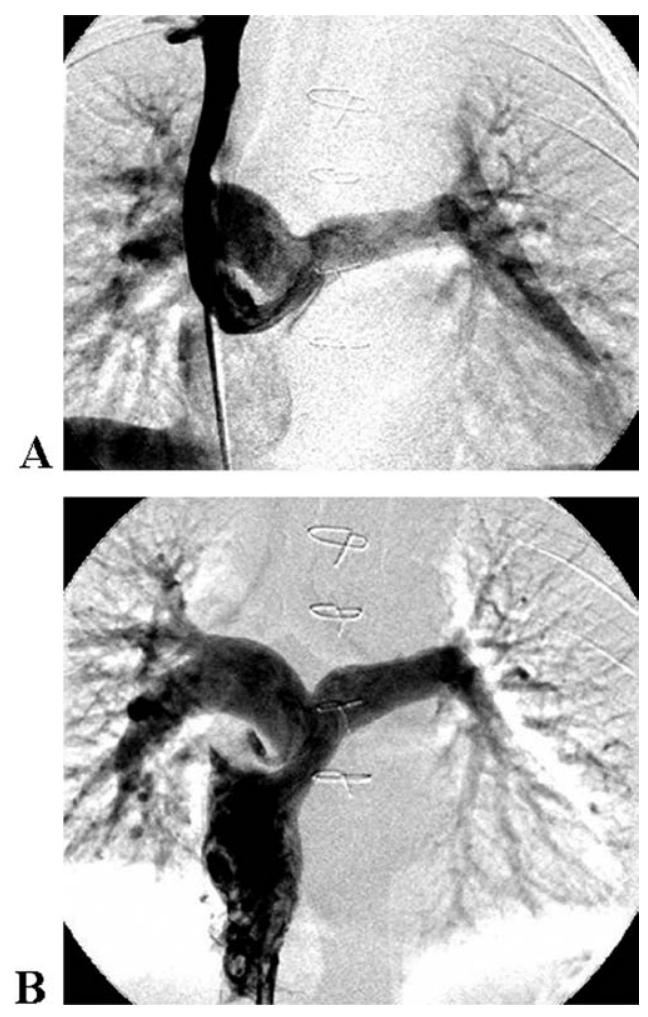

Figure 2. Postoperative angiogram: $A$, superior vena caval angiogram; $B$, inferior vena caval angiogram.

\section{Discussion}

The advantages of total cavopulmonary direct anastomosis have been a demonstrated reduction in the occurrence of turbulence and the prevalence and progression of early and late arrhythmia. ${ }^{2-4}$ Furthermore, there are all the advantages associated with the exclusive use of native tissue, including growth potential, as well as reduced risk of thrombus formation, elimination of peel formation, lower infection rate, and reduced cost. ${ }^{2-4}$

In addition to the above, our procedure has the advantage of a balanced PA and IVC flow distribution caused by the mixing route using the main PA and the possibility of attaining a good energy efficiency thanks to the merged connection of the superior and inferior venous flow.
In recent years, postoperative unresolved pulmonary arteriovenous malformations caused by an unbalanced IVC flow after TCPC have been reported. ${ }^{5}$ Although it has not been clarified at which degree of unbalanced IVC flow pulmonary atriovenous malformations will occur, it is very important to maintain a balanced IVC flow distribution after TCPC.

In conventional anatomic designs of TCPC, asymmetry of the connection between the SVC and the IVC has the advantage of a good energy efficiency but the disadvantage of an unbalanced IVC flow distribution, whereas the opposite is true in case of symmetry of the connection. Therefore directly bowed anastomosis with the SVC and IVC was performed for the merged connection of the superior and inferior venous flow, and the postoperative angiography and catheterization revealed smooth laminar mixing and low SVC and IVC pressures, respectively. Although further studies concerning the energy efficiency of this design are required, we believe that there are minimum energy losses caused by laminar mixing.

This procedure might be feasible in patients with an elongated SVC and well-developed main PA located to the right of or anteroposteriorly to the aorta. However, it is possible to construct this route by using autologous pericardium and the atrial wall, and we recently performed this designed autotissue TCPC using an atrial flap as the IVC route for a patient with pulmonary atresia and an intact ventricular septum.

\section{References}

1. Bove EL, de Leval MR, Migliavacca F, Guadagni G, Dubini G. Computational fluid dynamics in the evaluation of hemodynamics performance of cavopulmonary connections after the Norwood procedure for hypoplastic left heart syndrome. J Thorac Cardiovasc Surg. 2003;126: 1040-7.

2. Carotti A, Iorio FS, Amodeo A, et al. Total cavopulmonary direct anastomosis: a logical approach in selected patients. Ann Thorac Surg. 1993;56:963-4.

3. Van Son JAM, Reddy MV, Hanley FL. Extracardiac modification of the Fontan operation without use of prosthetic material. J Thorac Cardiovasc Surg. 1995;110:1766-8.

4. de Leval MR, Kilner P, Gewillig M, Bull C. Total cavopulmonary connection: a logical alternative to atriopulmonary connection for complex Fontan operations. Experimental studies and early clinical experience. J Thorac Cardiovasc Surg. 1988;96:682-95.

5. Ichikawa $\mathrm{H}$, Fukushima N, Ono M, et al. Resolution of pulmonary arteriovenous fistula by redirection of hepatic venous blood. Ann Thorac Surg. 2004;77:1825-7. 